\title{
NOD2-mediated dysbiosis predisposes mice to transmissible colitis and colorectal cancer
}

\author{
Aurélie Couturier-Maillard, ${ }^{1,2,3,4}$ Thomas Secher, ${ }^{5}$ Ateequr Rehman, 6 Sylvain Normand, ${ }^{1,2,3,4}$ \\ Adèle De Arcangelis, ${ }^{7}$ Robert Haesler, ${ }^{8}$ Ludovic Huot, 1,2,3,4 Teddy Grandjean, 1,2,3,4 \\ Aude Bressenot, ${ }^{9}$ Anne Delanoye-Crespin,, 1,2,3,4 Olivier Gaillot, 1,2,3,4 Stefan Schreiber, ${ }^{8}$ \\ Yves Lemoine, 1,2,3,4 Bernhard Ryffel,5,10 David Hot, 1,2,3,4 Gabriel Nùñez,11 \\ Grace Chen, ${ }^{11}$ Philip Rosenstiel, ${ }^{8}$ and Mathias Chamaillard ${ }^{1,2,3,4}$ \\ 1 University Lille Nord de France, Lille, France. ${ }^{2}$ Institut Pasteur de Lille, Center for Infection and Immunity of Lille, Lille, France. \\ ${ }^{3}$ Centre National de la Recherche Scientifique, Unité Mixte de Recherche, Lille, France. ${ }^{4}$ Institut National de la Santé et de la Recherche Médicale, \\ Lille, France. ${ }^{5}$ CNRS, UMR6218 IEM, Orléans, France. ${ }^{6}$ Universitätsklinikum Freiburg, Freiburg, Germany. \\ ${ }^{7}$ Institut de Génétique et de Biologie Moléculaire et Cellulaire, Department of Development and Stem Cells, Inserm U964, \\ CNRS UMR7104, Université de Strasbourg, Illkirch, France. ${ }^{8}$ Institute of Clinical Molecular Biology, University Hospital Schleswig-Holstein, \\ Campus Kiel, Kiel, Germany. Institut National de la Santé et de la Recherche Médicale, Vandoeuvre-lès-Nancy, France. \\ ${ }^{10}$ Institute of Infectious Disease \& Molecular Medicine, University of Cape Town, Cape Town, South Africa. ${ }^{11}$ University of Michigan, Ann Arbor, Michigan, USA.

\begin{abstract}
Instability in the composition of gut bacterial communities (dysbiosis) has been linked to common human intestinal disorders, such as Crohn's disease and colorectal cancer. Here, we show that dysbiosis caused by $\mathrm{Nod} 2$ deficiency gives rise to a reversible, communicable risk of colitis and colitis-associated carcinogenesis in mice. Loss of either Nod 2 or RIP2 resulted in a proinflammatory microenvironment that enhanced epithelial dysplasia following chemically induced injury. The condition could be improved by treatment with antibiotics or an anti-interleukin- 6 receptor-neutralizing antibody. Genotype-dependent disease risk was communicable via maternally transmitted microbiota in both Nod2-deficient and WT hosts. Furthermore, reciprocal microbiota transplantation reduced disease risk in Nod2-deficient mice and led to long-term changes in intestinal microbial communities. Conversely, disease risk was enhanced in WT hosts that were recolonized with dysbiotic fecal microbiota from Nod2-deficient mice. Thus, we demonstrated that licensing of dysbiotic microbiota is a critical component of disease risk. Our results demonstrate that NOD2 has an unexpected role in shaping a protective assembly of gut bacterial communities and suggest that manipulation of dysbiosis is a potential therapeutic approach in the treatment of human intestinal disorders.
\end{abstract}

\section{Introduction}

The gut flora is composed of a huge number of diverse microbial communities, within which Bacteroidetes, Firmicutes, and Proteobacteria are the dominant phyla $(1,2)$. In humans, the coexistence of resident commensals and a single layer of epithelial cells plays a beneficial role in regulating both energy harvesting from nutrients and immune system function $(3,4)$. In the absence of commensals, the colonic mucosa displays defects in goblet cell differentiation (5) and lymphoid tissue architecture (e.g., isolated lymphoid follicles) (6). Importantly, not all commensals are able to maintain quiescent, protective immunity (7). It has been hypothesized that disturbances of this balanced state (referred to herein as dysbiosis) directly or indirectly contribute to the pathogenesis of several immune-mediated intestinal illnesses, such as Crohn's disease (CD) and colitis-associated colorectal carcinoma (CAC).

$\mathrm{CD}$ is characterized by relapsing-remitting inflammatory responses that have a tendency to develop where the bacterial load is greatest. As such, short-term antibiotic treatment is used as a nonspecific therapy in some patients with CD (8). In particular, $\mathrm{CD}$ is associated with a greater preponderance of certain Bacte-

Authorship note: Aurélie Couturier-Maillard, Thomas Secher, and Ateequr Rehman contributed equally to this work. Philip Rosenstiel and Mathias Chamaillard are cosenior authors.

Conflict of interest: The authors have declared that no conflict of interest exists. Citation for this article: J Clin Invest. 2013;123(2):700-711. doi:10.1172/JCI62236. roidetes and a lower abundance of Firmicutes $(9,10)$. More importantly, subclinical dysbiosis has been observed in healthy relatives of patients with CD (11). However, it remains to be seen whether a defined, dysbiotic state is enough to trigger $\mathrm{CD}$, which in turn is a major risk factor for CAC (12). Epithelial dysplasia in this setting is thought to result from impaired intestinal barrier function and a proinflammatory milieu, which depends (at least partially) on the loss of bidirectional communication between a consortium of microorganisms and host molecules. However, the nature and mechanisms of these processes are entirely unknown. A clear understanding of the functional role of dysbiosis is thus needed in order to establish whether (a) a particular dysbiotic state is sufficient to instigate CD and/or CAC and (b) evaluate the potential for preventive manipulation of the gut microbiota in humans.

Although the nucleotide-binding oligomerization domaincontaining protein 2 (NOD2) is thought to play a decisive role in maintaining microbial tolerance at the intestinal barrier (13-16), its role in inducing innate and adaptive immunity is complex (17-19). The NOD2 protein is composed of a C-terminal leucine-rich repeat region, a centrally located nucleotide-binding oligomerization domain, and a N-terminal tandem of caspase recruitment domains that interact with the RIP2 adaptor within the cell (20). NOD2 is expressed by various cell populations within the colonic mucosa, including epithelial and myeloid cells. In mice, genetic ablation of NOD2 enhances the abundance of both ileal- and fecal-associated Bacteroidaceae (21-23). Likewise, the CD-associated NOD2 variant $\mathrm{L} 1007 \mathrm{fsinsC}$ is associated with 
enhanced mucosal colonization by the Bacteroidaceae in humans $(22,24)$. However, it remains to be seen whether the dysbiotic state caused by this CD-predisposing primary immunodeficiency is enough to instigate disease or is merely a consequence of the underlying disorder. This study is, to the best of our knowledge, the first to describe an unexpected role of NOD2 in the protection against intestinal inflammation and tumorigenesis afforded by gut bacterial communities in mice.

\section{Results}

Nod2/RIP2 deficiency confers a maternally transmissible colitis risk on immunocompetent hosts. Given that a stable composition of commensals is (at least in part) an inherited trait, we hypothesized that some of the missing heritability in CD could be explained by the transmission of a disease-predisposing bacterial community from one generation to another and between offspring. We therefore performed cohousing and cross-fostering experiments with Nod2- or RIP2-deficient mice and studied their characteristics in an experimentally induced colitis model that consists of either acute or chronic exposure to dextran sodium sulfate (DSS). Briefly, WT mice were cohoused with Nod2- or RIP2-deficient mice. Some animals were cohoused with mutant mice throughout the experiments (referred to as the $\mathrm{CH}-\mathrm{WT}_{\mathrm{Nod}_{2-/}}$ and $\mathrm{CH}-\mathrm{WT}_{\text {RIP2-/- }}$ groups), whereas others were separated from the mutants (referred to as the $\mathrm{SCH}_{-} \mathrm{WT}_{\text {Nod2-/- }}$ and $\mathrm{SCH}-\mathrm{WT}_{\text {RIP2-/- }}$ groups) just before disruption of the colonic barrier with DSS (Figure 1A). As a control, other WT mice were housed singly (referred to as the SH-WT group) prior to administration of DSS.

Unexpectedly, both CH-WT) (Figure 1, B and C) and separated cohoused WT (SCH-WT) (Figure 1D) mice showed a greater loss of body weight, more severe bloody diarrhea, and a higher histology score (Figure 1, E and F) after DSS exposure (relative to SH-WT animals). Likewise, mortality and morbidity (including weight loss, diarrhea, and rectal bleeding) were significantly enhanced during the recovery phase in DSS-treated, single-housed mutant mice (referred to as $\mathrm{SH}-\mathrm{Nod}_{2}{ }^{-/}$and $\mathrm{SH}-\mathrm{RIP}_{2}{ }^{--}$, respectively), when compared with similarly treated SH-WT animals (Supplemental Figure 1; supplemental material available online with this article; doi:10.1172/JCI62236DS1). These results show that the enhanced disease risk in CH-WT mice is more likely to result from inheritance of a disease-predisposing microbiota than from the more severe DSS-induced colitis observed in mutant mice. On the molecular level, secretion of the proinflammatory cytokine interleukin- 6 was transiently but significantly enhanced during the early phase of colitis in SH-Nod2 ${ }^{-/-}$animals, when compared with that in SH-WT animals (Supplemental Figure 2A). Furthermore, immunohistochemical analysis revealed the robust expression of interleukin-6 in dendritic-like cells and a low but detectable expression of interleukin- 6 in intestinal epithelial cells upon DSS challenge (Supplemental Figure 2B). In order to establish which infiltrating cells were expressing interleukin-6, we performed double immunofluorescence staining with anti-interleukin- 6 and anti-CD11b, anti-CD4, or anti-CD8a antibodies. In line with a previous report of significant interleukin-6 production by dendritic cells upon DSS challenge (25), none of the aforementioned subsets

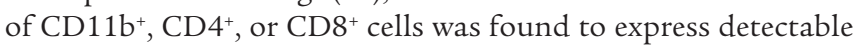
interleukin-6 in DSS-treated animals, regardless of the expression of RIP2 (Supplemental Figure 3). Since TNF- $\alpha$ is also a key regulator of intestinal inflammation and tumorigenesis in $\mathrm{T}-\mathrm{bet}^{t^{--}} \mathrm{Rag}^{-/-}$ ulcerative colitis (TRUC) mice in response to bacterial commensals
(26), we next quantified protein levels of TNF- $\alpha$. In contrast to the results in the literature for TRUC mice, we failed to detect any RIP2-dependent changes of TNF- $\alpha$ secretion over time (Supplemental Figure 7B, respectively). Likewise, DSS-treated SH-WT and $\mathrm{SH}-\mathrm{Nod} 2^{--}$animals had similar colonic levels of Tnfa gene transcripts (Supplemental Figure 2C). In contrast, qRT-PCR analysis confirmed a transient but significant elevation of transcript levels for not only the gene encoding interleukin-6 (Il6) but also for its known target genes, including those encoding matrix metallopeptidase 13 (Mmp13), prostaglandin-endoperoxide synthase 2 (Ptgs2), and secreted phosphoprotein 1 (Spp1, also known as early T lymphocyte activation 1) (Supplemental Figure 2D). Moreover, expression of $\operatorname{Reg} 3 b$ and $\operatorname{Reg} 3 g$ (which are also linked to the interleukin-6mediated signaling pathway; refs. 27,28 ) was downregulated in Nod2 $2^{-1-}$ mice upon DSS treatment (Supplemental Figure 2D). As observed in $\mathrm{CH}-\mathrm{WT}_{\mathrm{RIP2-}-\mathrm{-}}$ mice, body weight loss (Figure $2 \mathrm{~A}$ ) and colon shortening (Figure $2 \mathrm{~B}$ ) in WT animals reared with a RIP2 $2^{-/}$mother since birth (referred to as CF-WT $\mathrm{WIP2-/}_{\text {- }}$ animals) were more severe than those in cross-fostered WT (CF-WT) progeny mice. Accordingly, microscopic examination of H\&E- and PAS-stained colonic sections revealed greater histopathological changes (including colon crypt loss, immune cell influx, and ulcer-

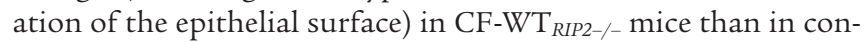
trols (Figure 2C). This result suggested that the vulnerability to colitis transmitted from one generation of mutant mice to another may be due to inheritance of a dysbiotic bacterial flora that sensitizes the colonic mucosa to chemical insult.

Fecal dysbiosis in Nod2-deficient mice sensitizes the colonic mucosa to chemical injury. We next sought to establish whether or not the disease-predisposing microbiota in Nod2-deficient mice directly triggered spontaneous inflammatory response in WT animals prior to any injury via DSS. To this end, WT animals were single-housed or cohoused for 4 weeks with either Nod2- or RIP2-deficient mice. Gene expression analysis was performed on RNA extracted from the isolated colons of $\mathrm{SH}-\mathrm{WT}$ and $\mathrm{CH}-\mathrm{WT}_{\mathrm{Nod}_{2-1}-}$ animals that had not been treated by DSS (Supplemental Figure 4 and Supplemental Table 1). In contrast to what had been observed during DSS-induced colitis, whole-genome expression analysis revealed similar levels of transcription of genes involved in the inflammatory response (including Il6, Mmp13, Ptgs2, and Spp1) in SH-WT and $\mathrm{CH}-\mathrm{WT}_{\mathrm{Nod2-}-\mathrm{-}}$ animals. Accordingly, we failed to observe any NOD2-dependent differences in either Il6 or Tnfa gene expression between germ-free (GF) and specific pathogen-free (SPF) mice prior to any injury (Supplemental Figure 5). More importantly, a qRT-PCR analysis confirmed the differential gene expression of claudin 5 (Cldn5) in $\mathrm{CH}-\mathrm{WT}_{\mathrm{Nod} 2-/-}$ and $\mathrm{CH}-\mathrm{WT}_{\mathrm{RIP2-} /-}$ animals (relative to that of single-housed control animals) prior to DSS exposure (Figure 3A). Likewise, levels of Cldn5 transcript were found to be upregulated in animals at risk of disease upon DSS injury, i.e., the $\mathrm{SCH}_{-} \mathrm{WT}_{\text {Nod2-/- }}$ (Figure $3 \mathrm{~B}$ ) and $\mathrm{CF}-\mathrm{WT}_{\text {RIP2-/- }}$ animals (Figure 3C). Furthermore, gene ontology analyses revealed an increase in the transcription of genes whose products are involved in apoptosis, necrosis, and tumorigenesis (Supplemental Figure 4 and Supplemental Table 2). Taken as a whole, our results suggest that response to components of the microbiota from either Nod2or RIP2-deficient mice did not result in spontaneous colitis. It is worth noting that NOD2-mediated dysbiosis impaired coordination of the expression of molecules required for integrity of the colonic epithelium that would lead to enhanced intestinal inflammation in response to injury. 
A

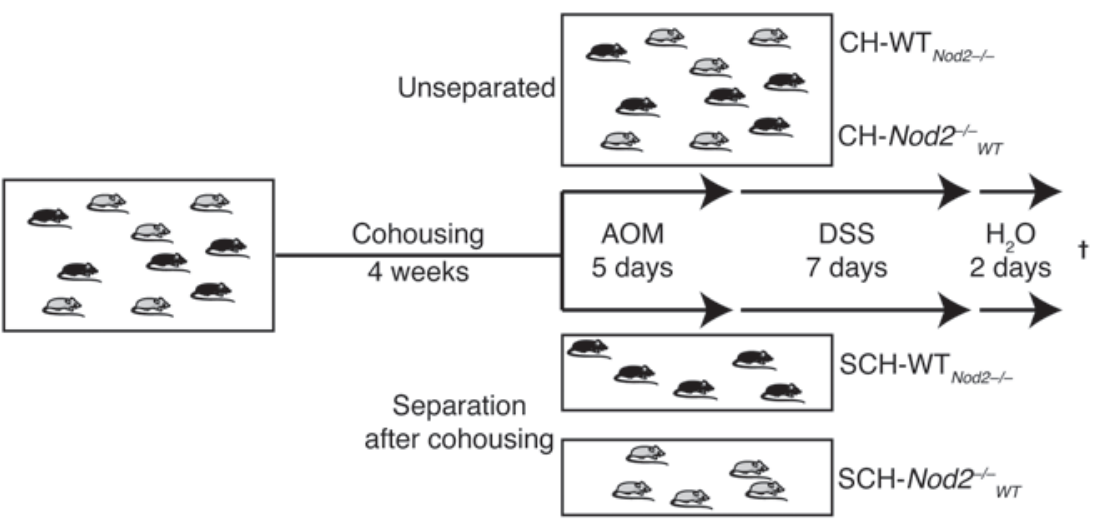

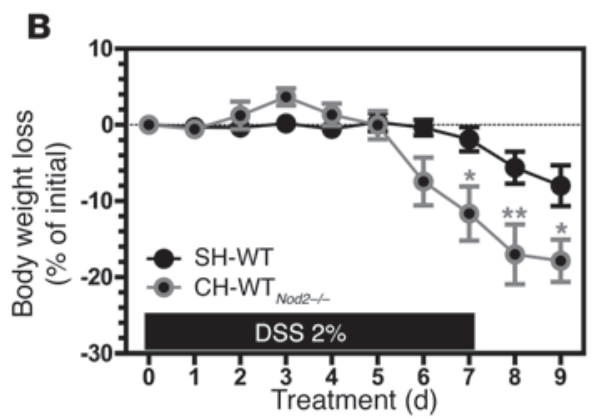

\section{C}
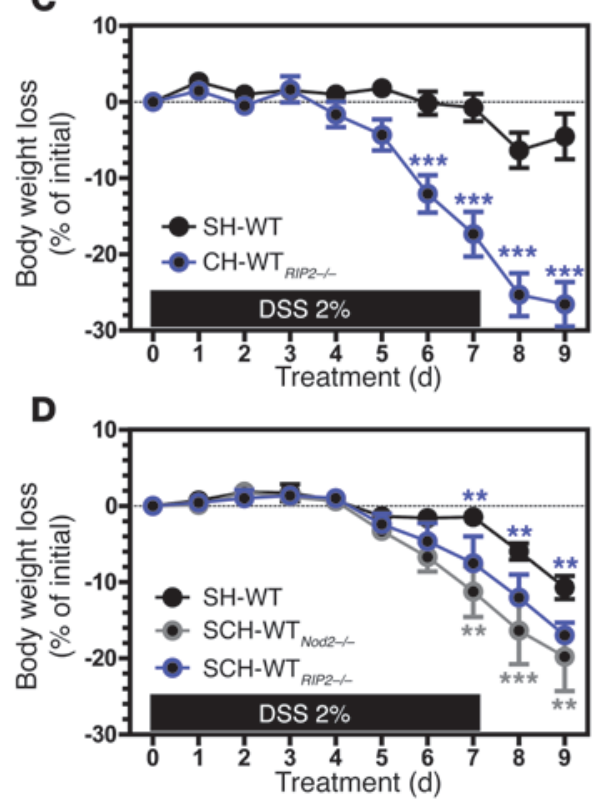

E

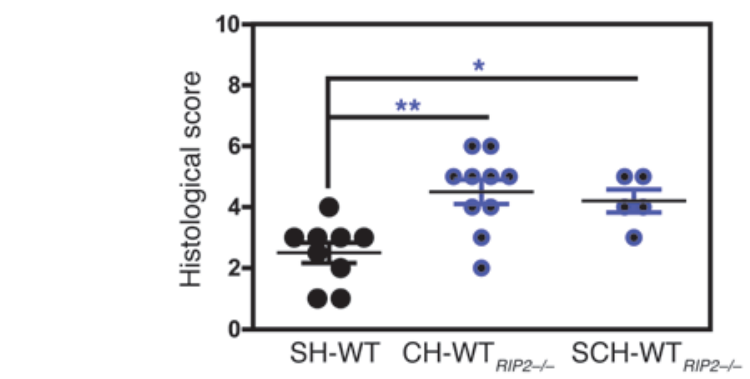

$\mathbf{F}$
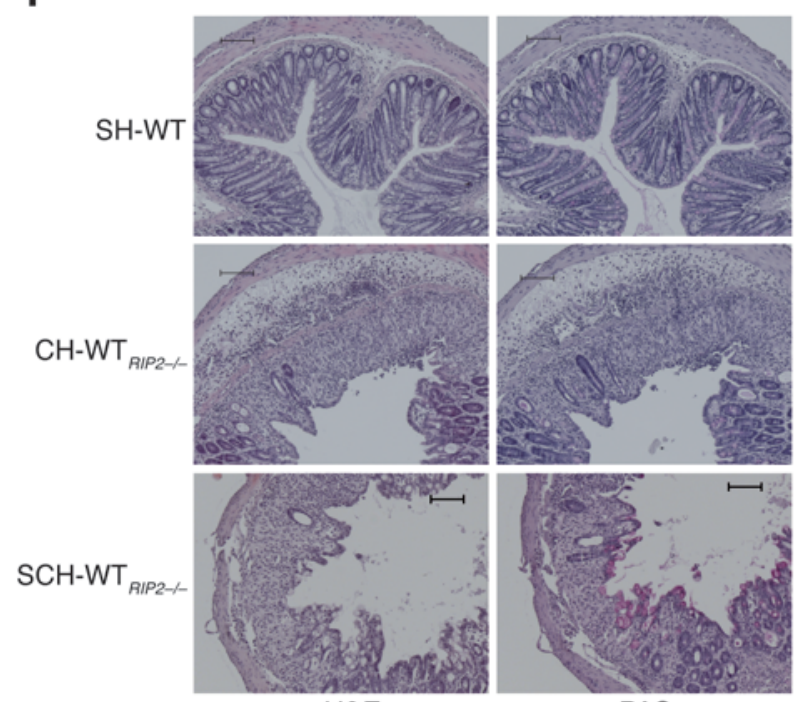

H\&E

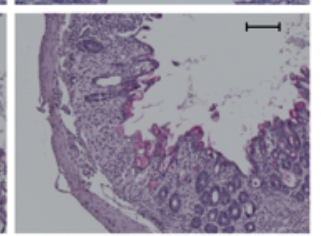

PAS

Figure 1

The absence of NOD2/RIP2 confers a transmissible risk for colitis, even to immunocompetent hosts. (A) Five days after AOM administration

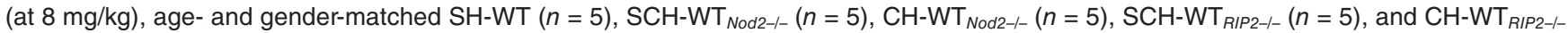
$(n=4)$ mice received ad libitum 2\% (w/v) DSS (molecular mass, 35-40 kDA; TdB Consultancy) for 7 days followed by 2 days of regular drinking water. Mutant and WT animals were cohoused at a 3:2 or 1:1 ratio on the same diet for a 4-week period. WT cohoused mice were either left with or separated from mutant mice before being challenged by 1 cycle of DSS-AOM. The cross indicates necropsy. Changes in body weight of

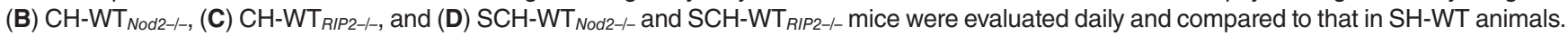
(E) Histological scoring was determined at day 9. (F) Representative H\&E and PAS staining of paraffin-embedded longitudinal sections from colons of SH-WT, SCH-WT RIP2-/, and CH-WT RIP2-/ mice at day 9. Scale bar: $100 \mu \mathrm{m} .{ }^{*} P<0.05 ;{ }^{* \star} P<0.01 ;{ }^{* * *} P<0.001$. 
A

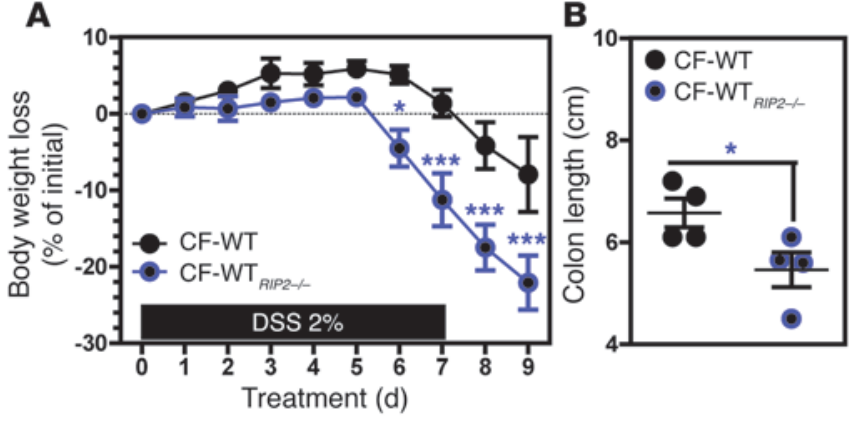

C
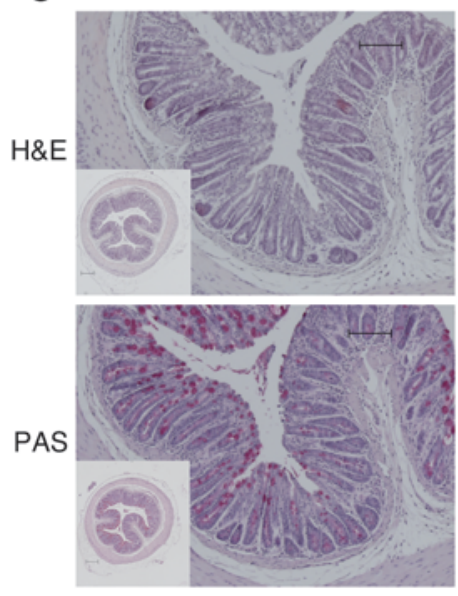

CF-WT
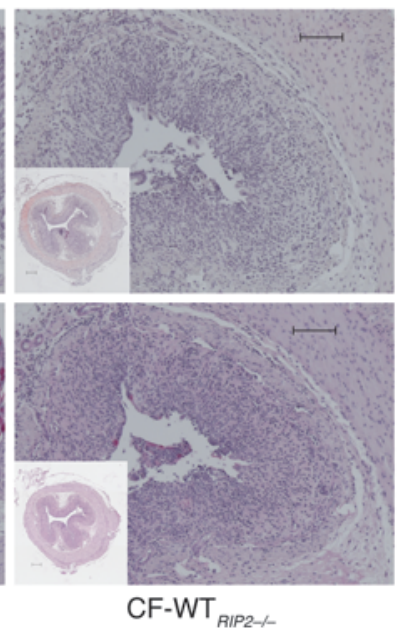

A communicable risk of colitis-associated colorectal cancer in the absence of Nod2 and RIP2. Colorectal carcinogenesis is a redoubtable complication of CD in humans. It is thought to result from a complex, microbial-driven inflammatory response (29). Furthermore, mutations in NOD2 have been shown to predispose to both colorectal cancer and gastric lymphoma in humans $(30,31)$. We therefore sought to establish whether or not the communicable risk of colitis in $\mathrm{Nod2}^{-/-}$and $\mathrm{RIP} 2^{-/-}$mice influenced the development of CAC in CH-WT mice. To this end, single-housed and cohoused mice were studied in a well-characterized experimental model of CAC (32). Under these conditions, the tumor load in the distal gut of both $\mathrm{CH}-\mathrm{WT}_{\mathrm{Nod2-}-\mathrm{-}}$ and $\mathrm{CH}-\mathrm{WT}_{\mathrm{RIP2-}-\text { - }}$ mice was greater than that in SH-WT animals (Figure 4, A-C). Similarly, a lack of NOD2 or RIP2 was associated with greater CAC-related mortality and morbidity in two independent SPF environments (Figure 4, D and E, and Supplemental Figure 6, A and B). Moreover, the greater tumor burden (Figure 4, D and E) and

\section{Figure 2}

The risk for colitis in RIP2-deficient mice is maternally transmissible. WT animals were reared with either a RIP2-deficient or -sufficient mother since birth $(n=4)$. Five-week-old cross-fostered mice were then exposed to 1 cycle of DSS-AOM, as described earlier. Changes in (A) body weight and (B) colon length were evaluated daily. (C) Representative H\&E and PAS staining of paraffin-embedded transversal sections from colons of CF-WT and CF-RIP2-/- mice at day 9. CF refers to cross-fostered animals. Scale bar: $100 \mu \mathrm{m}$. ${ }^{\star} P<0.05$; ${ }^{* \star \star} P<0.001$.

growth (Figure 4F) observed in $\mathrm{SH}-\mathrm{Nod}_{2}{ }^{-/}$mice were preceded by enhanced epithelial proliferation, as determined by BrdU incorporation in vivo (Figure 4G). As observed following DSS treatment, interleukin-6 secretion was significantly enhanced during the early phase of tumor initiation but not during tumor progression in $\mathrm{CH}_{-} \mathrm{WT}_{\mathrm{RIP2-} /-}$ animals (relative to that in $\mathrm{SH}-\mathrm{WT}$ animals) (Supplemental Figure 7). Taken as a whole, these results demonstrate that NOD2 or RIP2 deficiency was associated with the presence of a maternally transmissible set of microorganisms that appeared to sensitize the intestinal mucosa to both colitis and CAC - even in a nongenetically predisposed host.

Broad-spectrum antibiotic treatment reduces disease severity, bacterial counts, inflammatory response, and immune cell trafficking in Nod2-deficient mice upon injury. The unexpected transmission of disease-predisposing microorganisms in mice lacking NOD2 or RIP2 prompted us to wonder whether antibiotic treatment would limit the development of dysplasia in a proinflammatory colonic environment. Following combination therapy with broad-spectrum antibiotics, we found that the genotype-driven morbidity and mortality were markedly lower than those in untreated animals. It is noteworthy that the disease was also less severe in antibiotic-treated WT animals (Figure 5A). These observations indicate a more general role for bacterial commensals in the pathogenesis of colitis and CAC in the absence of NOD2 or RIP2. In Nod2-deficient animals, the changes in gut microbiota after antibiotic treatment were associated with a significantly lower body weight loss (Figure 5A), a lower Bacteroides count (Figure 5B), and decreased recruitment of CD11 b $\mathrm{Ly}^{+} 6 \mathrm{G}^{+}$ granulocytic cells within the colonic mucosa (Figure 5C) compared with that in untreated animals. In contrast, we failed to observe any changes in the influx of either CD $11 \mathrm{~b}^{-} \mathrm{CD} 11 \mathrm{c}^{+}$or $\mathrm{CD} 11 \mathrm{~b}^{+} \mathrm{CD} 11 \mathrm{c}^{+} \mathrm{IA} / \mathrm{IE}^{\mathrm{hi}}$ macrophages (Figure 5, D and E). To further characterize the disease remission induced by antibiotic treatment in both WT and Nod2-deficient mice, we next isolated RNA from the colon after 1 cycle of DSS-azoxymethane (DSS-AOM) exposure. A genome-wide expression analysis and a gene ontology analysis revealed that antibiotic treatment influ-

\section{Figure 3}

Enhanced expression of claudin 5 in response to dysbiosis coupled to NOD2 deficiency. WT animals were either single housed $(n=5)$ or cohoused at a 1:1 ratio with Nod2-deficient mice $(n=5)$ on the same diet for a 4-week period. qRT-PCR analysis was performed on extracted RNAs from the colons of (A) CH-WT, (B) SCH-WT, and (C) CF-WT animals as well as SH-WT animals. Mean \pm SEM. ${ }^{*} P<0.05$; ${ }^{* *} P<0.01$. $\mathrm{SCH}, \mathrm{CH}$, and $\mathrm{SH}$ refer to separated cohoused, cohoused, and singlehoused animals, respectively.
A

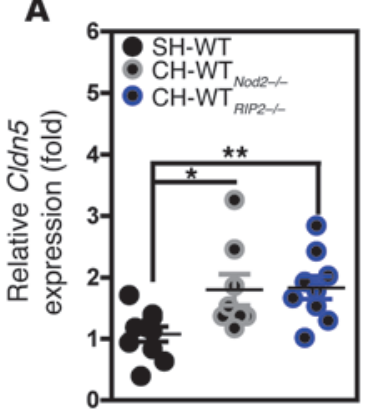

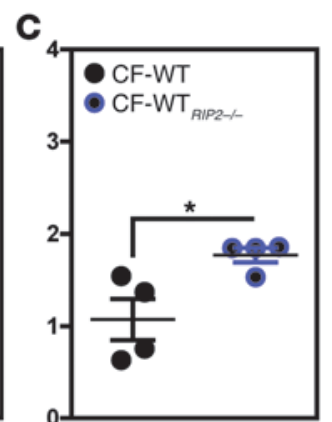


A

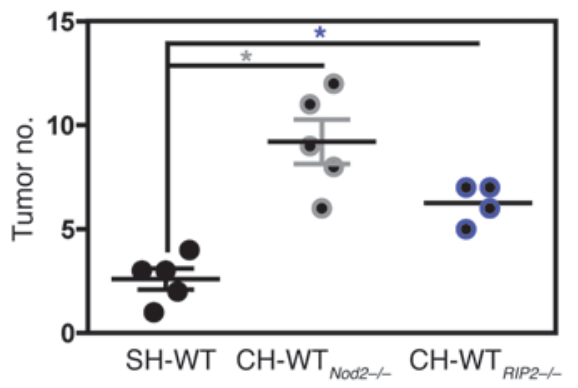

B

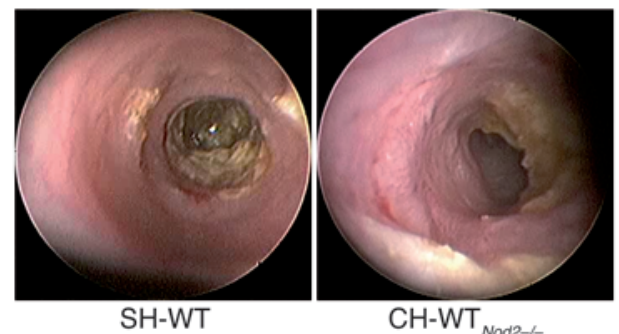

C

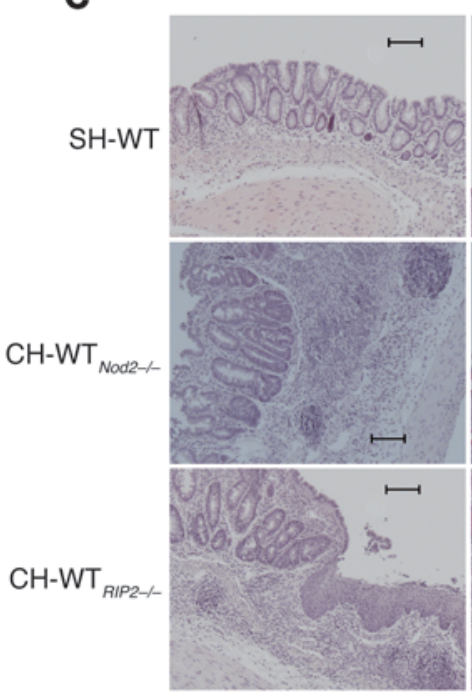

$H \& E$

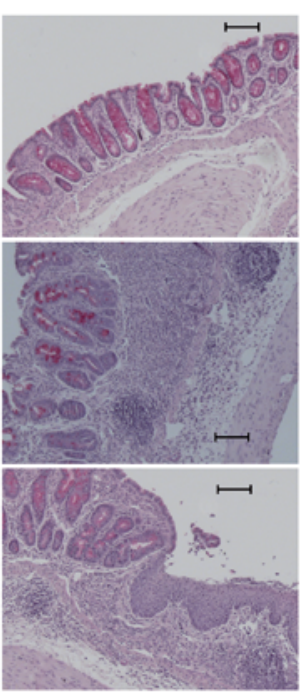

PAS

D

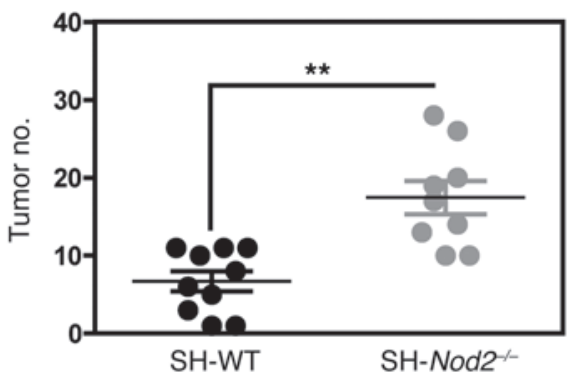

E

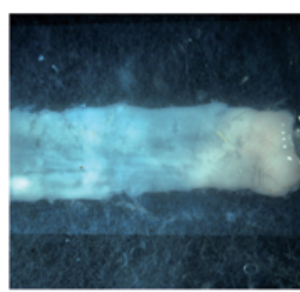

SH-WT

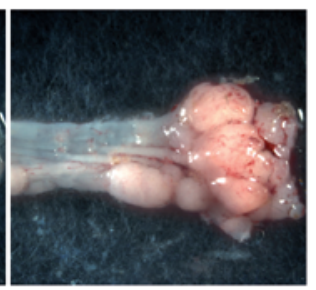

SH-Nod2 ${ }^{-1}$

$\mathbf{F}$

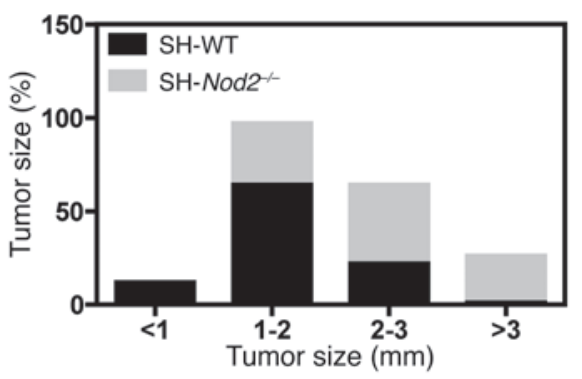

G

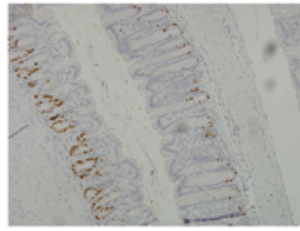

SH-WT

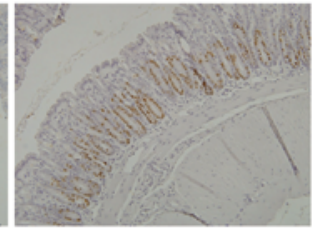

$\mathrm{SH}-\mathrm{Nod}_{2}$

\section{Figure 4}

NOD2-driven risk for colitis-associated colorectal cancer is communicable to WT host. Five days after AOM administration (at $8 \mathrm{mg} / \mathrm{kg}$ ), SH-WT,

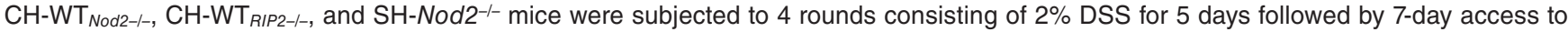
regular drinking water. (A) Macroscopic and (B) endoscopic quantification of colonic tumor burden in SH-WT $(n=5)$, CH-WT Nod2-- $(n=5)$, and

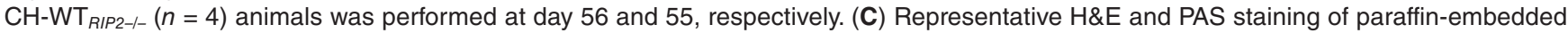
transversal sections from colons of SH-WT, CH-WT Nod2-- $_{\text {- }}$ and $\mathrm{CH}_{-}-\mathrm{WT}_{\text {RIP2-/- }}$ mice at day 56. Scale bar: 100 um (D) Macroscopic quantification of colonic tumor burden in SH-WT $(n=10)$ and SH-Nod2--- $(n=10)$ animals was performed at day 56. (E) Representative photographs of dissected colons of SH-WT and SH-Nod2 ${ }^{-/-}$mice at day 56. (F) Macroscopic quantification of colonic tumor growth in SH-WT and SH-Nod2 ${ }^{-/-}$animals was performed at day 56. (G) BrdU incorporation in vivo (original magnification, $\times 200$ ). Mean \pm SEM. ${ }^{*} P<0.05 ;{ }^{* *} P<0.01$.

enced the expression of 56 genes primarily involved in infiltration of neutrophils and cell movement of granulocytes and myeloid cells (Supplemental Figure 8). In line with previous findings in DSS-treated animals, qRT-PCR analysis confirmed that the enhanced transcription of Il6, Ptgs2, and Spp1 in diseased Nod2-deficient mice returned to normal levels after antibiotic treatment, when compared with similarly treated WT animals (Supplemental Tables 3 and 4 and Supplemental Figure 9). Last, tumor growth (as determined by the weight-to-length ratio) was significantly less rapid in antibiotic-treated mice - regardless of the genotype (Figure 5F). Overall, our results suggest that certain bacterial commensals instigate (either individually or together) an exaggerated inflammatory response that favors intestinal tumorigenesis upon injury.

Enhanced interleukin-6 secretion is required for tumor growth in Nod2and RIP2-deficient mice. In line with previous reports $(33,34)$, we next sought to determine whether or not an enhanced, early interleukin-6-mediated response to injury contributes to intes- 
A

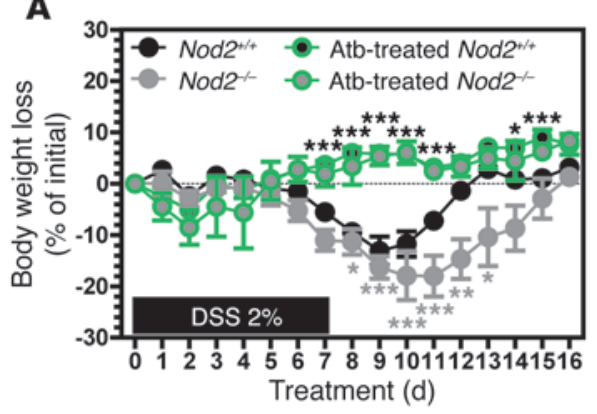

C
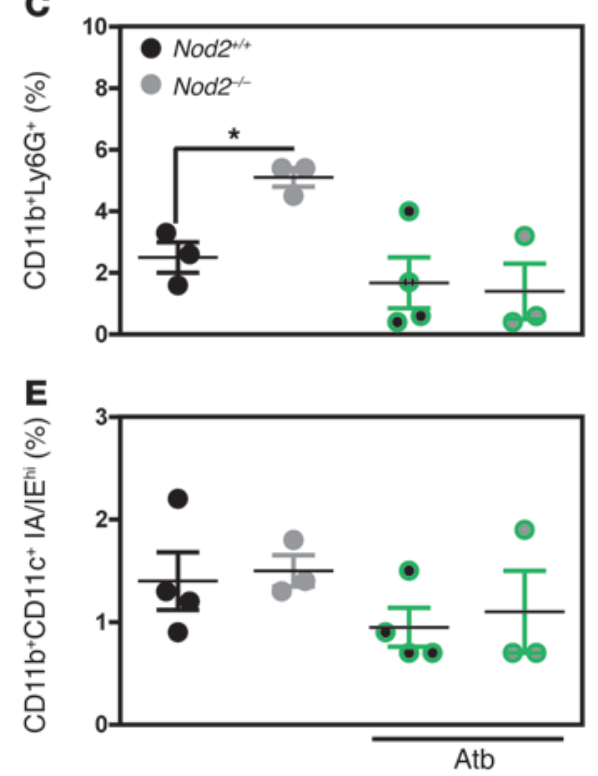

B

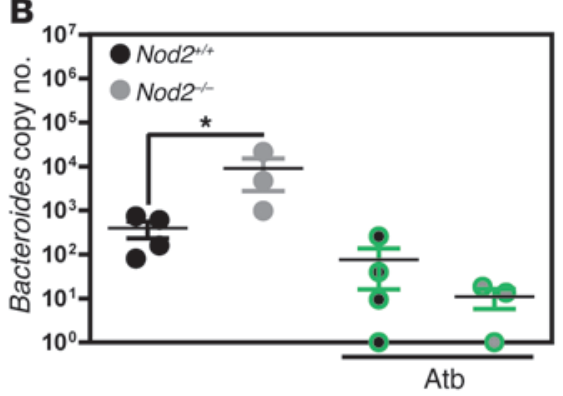

D
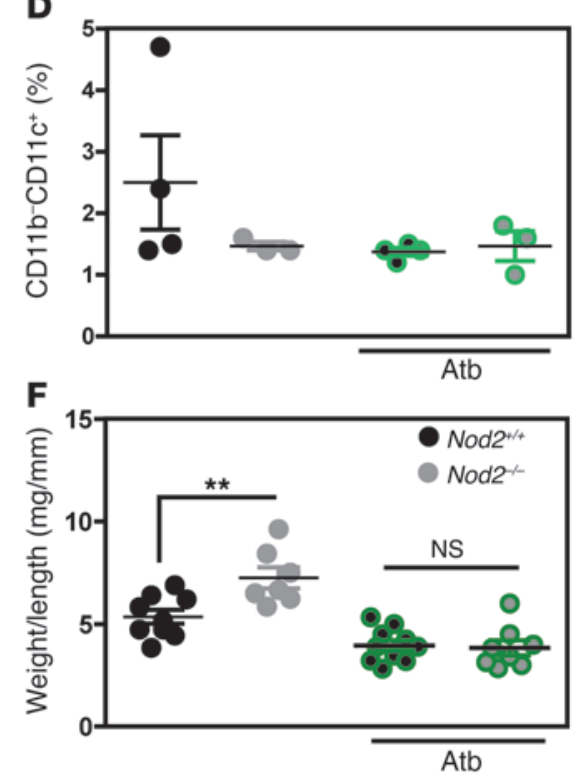

\section{Figure 5}

Broad-spectrum antibiotic treatment improves intestinal inflammation and tumorigenesis in Nod2-deficient mice. Five days after AOM administration (at $12 \mathrm{mg} / \mathrm{kg}), \mathrm{Nod}_{2+/+}(n=4)$, Nod2-/$(n=3)$, antibiotic-treated (Atb-treated) WT $(n=4)$, and Atb-treated Nod2-/$(n=3)$ mice were subjected to $2 \%$ DSS for 7 days followed by 9 -day access to regular drinking water. (A) Changes in body weight were monitored daily. (B) Colonic-associated Bacteroides load was determined by specific q-PCR analysis at day 16. (C-E) Ly6G, IA/IE, CD11c, and CD11b staining of the lamina propria cell population in Nod2 $2^{-/-}$ mice treated or not with antibiotics as indicated was quantified by FACS analysis. (F) Colon weight/length ratio quantification on $\mathrm{Nod}_{2}^{+/+}(n=10)$, Nod2 ${ }^{-/-}$ $(n=9)$, antibiotic-treated $\mathrm{Nod}^{+/+}(n=10)$ and antibiotic-treated Nod2 ${ }^{--}(n=10)$ mice was performed at day 58. ${ }^{*} P<0.05$; ${ }^{* *} P<0.01 ;{ }^{* *} P<0.001$. tinal tumorigenesis. A week before the last cycle of DSS-AOM, Nod2 $2^{-/}$mice were given a weekly, i.p. injection of a monoclonal anti-interleukin- 6 receptor antibody that reportedly suppresses Th1 cell-mediated colitis in mice (35). Endoscopic (Figure 6A) and macroscopic (Figure $6 \mathrm{~B}$ ) evaluations revealed that anti-interleukin- 6 receptor treatment resulted in a significant decrease in tumor burden, when compared with untreated mutant animals and regardless of the genotype. Accordingly, interleukin- 6 receptor neutralization in $\mathrm{Nod}^{-/-}$mice resulted in the normalization of transcript levels for the following antibiotic-sensitive genes: Il6, Ptgs2, Spp1, Reg3g, and Reg3b (Figure 6C). This agrees with previous reports in which genetic ablation of $I l 6$ reduced tumor load and growth (28) and interleukin- 6 hyperactivation promoted tumor progression $(33,34)$.

The interleukin-6-mediated control of disease progression is linked to changes in microbial ecology. We next looked at whether disease progression was linked to specific, NOD2-dependent changes in the relationship between bacterial communities and interleukin-6mediated signaling pathways. A culture-independent analysis of the mucosal-associated microbiota was performed by multiplex pyrosequencing of V1-V2 region amplicons of bacterial 16S rRNA genes. The respective impacts of host genotype, treatment, and intestinal tumorigenesis on the composition and structure of the microbial communities were assessed in a multivariate principal coordinate analysis (PCoA) and a canonical correspondence analysis (CCA). The PCoA plots of the UniFrac distances revealed a clear separation between genotypes in untreated animals but not after anti-interleukin-6 receptor treatment (Figure 7A). A CCA was used to detect interactions between bacterial composition (at the genus level) on one hand and factors such as intestinal tumorigenesis, genotype, and anti-interleukin- 6 receptor treatment on the other. The analysis identified NOD2 genotype and anti-interleukin- 6 receptor treatment as major contributors to the observed changes in the composition of mucosal-associated bacterial communities (Figure 7B). Notably, we observed changes in the abundance of commensals related to the Bacteroidetes phyla (Figure 7C) that (according to a similarity percentage [SIMPER] analysis) included Rikenella and Paludibacter (Figure 7D). Notably, anti-interleukin- 6 receptor treatment of mice lacking NOD2 was associated with a greater abundance of Rikenella $(P<0.02)$, suggesting that protective assembly of the microbiota may result from a specific gene-plus-treatment interaction. In contrast, CCA revealed that dysbiosis only made a minor contribution to tumor growth (Figure 7B). Hierarchical clustering of species-level operational taxonomic units (OTUs) was performed using ranked, normalized data (Supplemental Figure 10). Following anti-interleukin- 6 receptor treatment, the consortium of mucosal-associated bacteria was more diverse in both tumoral and chronically inflamed colonic mucosae (Figure 7, E and F). Collectively, these results suggest that neutralization of the interleukin-6-mediated signaling pathway restores complex, NOD2-dependent control of the colonic-associated microbiota in mice. 
A

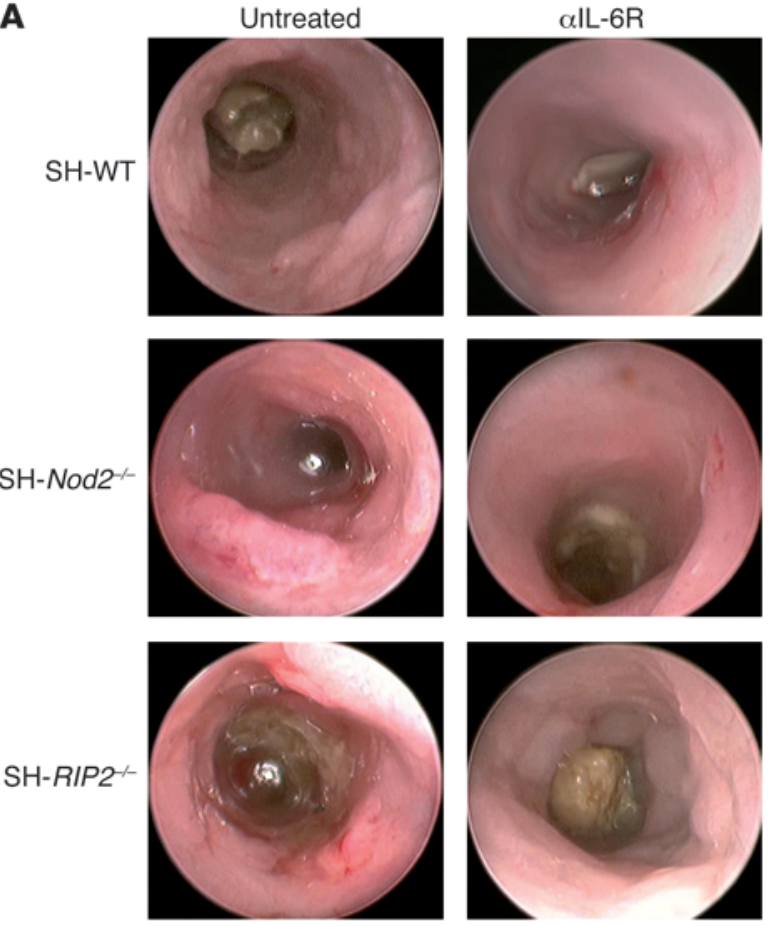

B

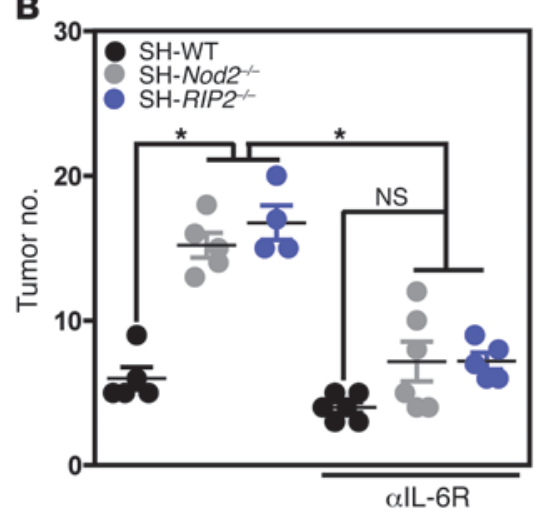

C

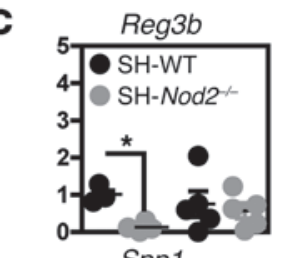

Spp1

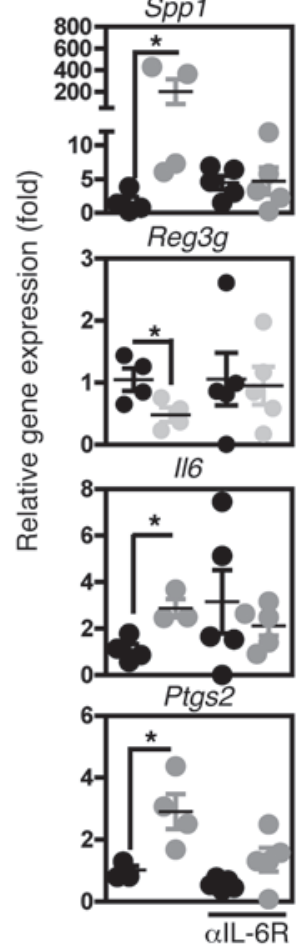

Figure 6

Neutralizing interleukin-6 reduces tumor progression in Nod2- and RIP2-deficient mice. Five days after AOM administration (at $8 \mathrm{mg} / \mathrm{kg}$ ) SH-WT $(n=5)$, SH-Nod2--- $(n=5)$, and SH-RIP2--- $(n=4)$ mice were exposed to 4 cycles of DSS, which consisted of 5 days separated by a 7-day period of regular water. An interleukin-6 receptor antibody ( $\alpha \mathrm{IL}-6 \mathrm{R})$ (1 mg per mouse i.p.) was given once a week from day 31 to 56 or not to AOMexposed mice. (A) Endoscopic and (B) macroscopic evaluation of mice was performed at day 55 and 56, respectively. (C) Colonic transcript levels of Reg3b, Reg3g, II6, Ptgs2, and Spp1 in untreated mice $(n=4)$ and anti-interleukin-6 receptor-treated $(n=4)$ animals are shown as determined by $q-P C R$ evaluation at day $56 .{ }^{*} P<0.05$.

The colitis risk in Nod2-deficient mice is mitigated by fecal transplantation. Given that the risk of colitis in $\mathrm{Nod2}^{-/-}$mice was communicable to Nod2-sufficient animals (Figure 1), we sought to formally determine whether the combination of fecal dysbiosis and NOD2 deficiency intrinsically conferred a risk of disease - even in the absence of any signs of inflammation. After 4 weeks of colonization with a $0.2 \mathrm{ml}$ supernatant of fresh fecal homogenate from either nondiseased $\mathrm{Nod}^{-\gamma_{-}}$or $\mathrm{Nod}^{+/+}$mice that were reared under SPF conditions, recolonized GF WT $\left(\mathrm{GF}-\mathrm{Nod}^{+/+}\right)$mice were challenged with a single DSS-AOM cycle. Under these conditions, the weight loss and histopathology scores of $\mathrm{Nod}^{+/+}$mice recolonized with fecal flora from $\mathrm{Nod} 2^{-/-}$mice (referred to as $\mathrm{Nod}^{-/-} \rightarrow \mathrm{GF}-\mathrm{Nod} 2^{+/+}$mice) were significantly greater than those recorded in GF-Nod2 $2^{+/+}$mice recolonized with fecal flora from $\mathrm{Nod} 2^{+/+}$mice (referred to as $\mathrm{Nod} 2^{+/+} \rightarrow \mathrm{GF}-$ $\mathrm{Nod} 2^{+/+}$mice) (Figure 8A). Another characteristic lesion of DSS-induced colitis is the loss of mucin-producing epithelial cells, which are usually revealed with $\mathrm{PAS}^{+}$staining. Only recipients colonized with fecal microbiota from $\mathrm{Nod}^{-/-}$mice lacked $\mathrm{PAS}^{+}$staining (Figure 8, $\mathrm{B}$ and $\mathrm{E})$; this finding suggests that the fecal microbiota of $\mathrm{Nod}_{2-1}^{-1}$ mice intrinsically contribute to the disease risk. We next sought to restore the ecological balance in the intestinal microbiome in $\mathrm{Nod}^{-/-}$ mice and establish whether or not this would decrease the host's vulnerability to colonic injury. GF-Nod2 $2^{-/}$mice were generated and then colonized with $0.2 \mathrm{ml}$ of the supernatant of a fresh fecal homogenate from either SPF $\mathrm{Nod}^{-/-}$or SPF $\mathrm{Nod}_{2}^{+/+}$mice. As shown in Figure 8, D-F, body weight loss, tissue erosion, and immune cell influx were lower in $\mathrm{Nod}_{2}{ }^{++} \rightarrow \mathrm{GF}-\mathrm{Nod2} 2^{-/-}$mice than in similarly treated $\mathrm{Nod} 2^{-/-} \rightarrow \mathrm{GF}-\mathrm{Nod} 2^{-/-}$animals. PCoA plots highlighted the differences in microbial composition and structure between recipients with a higher risk of colitis ( $\mathrm{Nod2}^{-/-} \rightarrow \mathrm{GF}-\mathrm{Nod}^{-/-}$and $\mathrm{Nod2^{-/- } \rightarrow \mathrm { GF } -}$ $\mathrm{Nod}^{+/+}$animals) and those with a lower risk of colitis (Figure 8G). It is noteworthy that both fecal- and mucosal-associated phylodiversity in both $\mathrm{Nod}_{2--} \rightarrow \mathrm{GF}-\mathrm{Nod} 2^{-/-}$and $\mathrm{Nod}_{2-2^{--} \rightarrow \mathrm{GF}-\mathrm{Nod} 2^{+/+}}$mice was markedly lower than that in $\mathrm{Nod}_{2}{ }^{+/+} \rightarrow \mathrm{GF}-\mathrm{Nod}_{2} 2^{--}$mice $(P<0.01$; Supplemental Figure 11A and Supplemental Figure 12A). Furthermore, a SIMPER analysis revealed that Bacteroides, Escherichia/Shigella, Robinsoniella, Paludibacter, Butyrivibrio, and Lachnobacterium were the genera that contributed to at least $5 \%$ of the shifts in bacterial communities, when comparing colitis-vulnerable and colitis-resistant recipients. Furthermore, Nod2 $2^{-/-} \rightarrow \mathrm{GF}-\mathrm{Nod}_{2}{ }^{--}$and $\mathrm{Nod} 2^{-/-} \rightarrow \mathrm{GF}-$ 
A

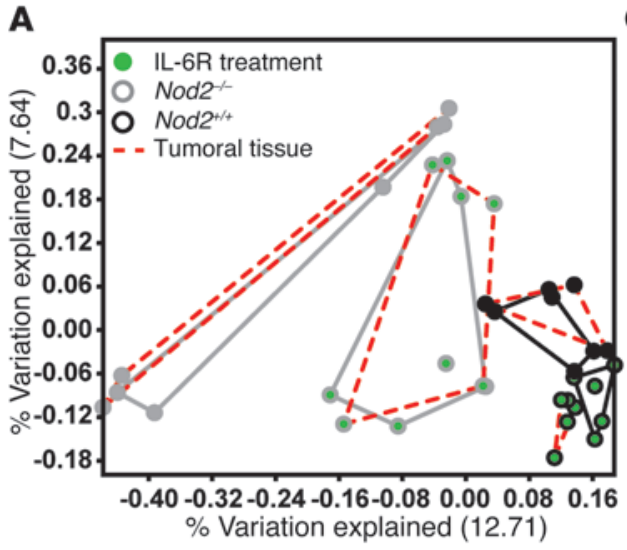

c

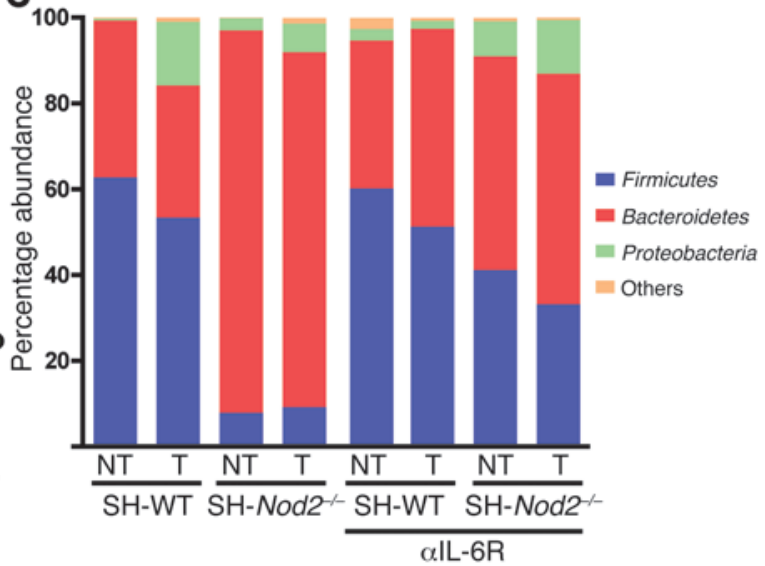

$\mathrm{D}$

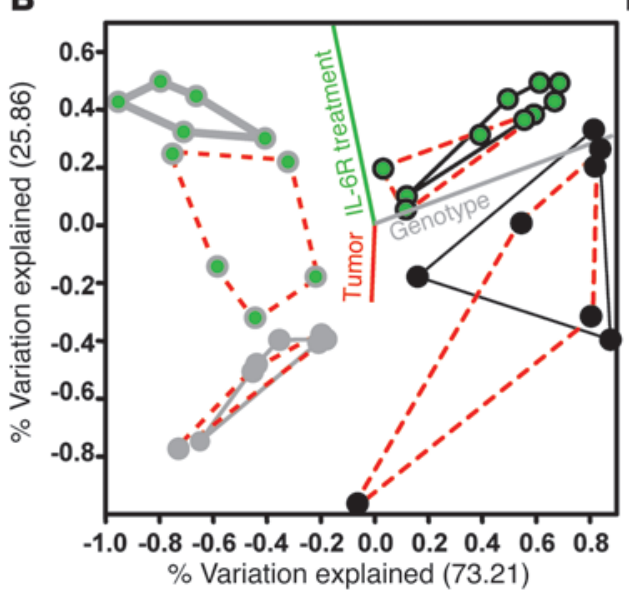

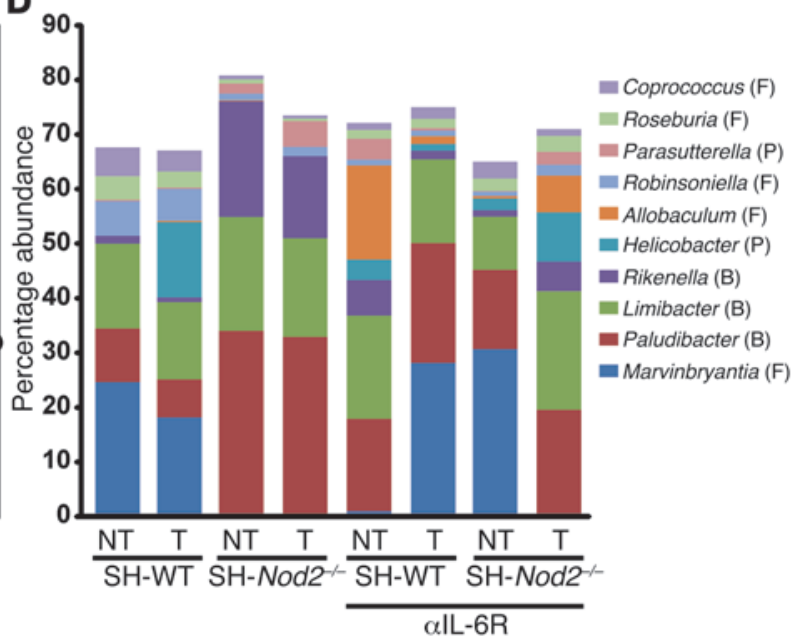

E

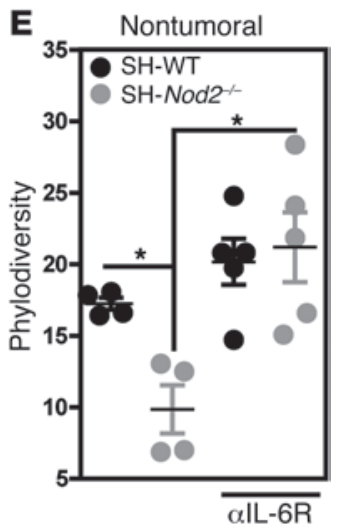

$\mathbf{F}$

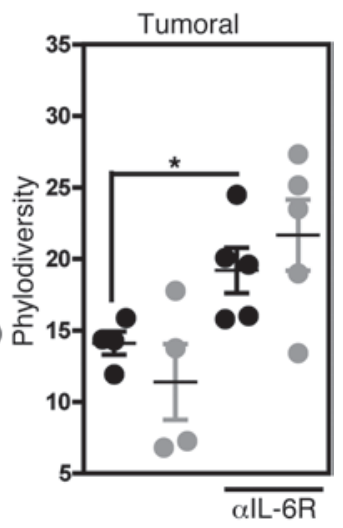

Figure 7

Interleukin-6-mediated control of disease progression is linked to NOD2-driven dysbiosis in an experimental model of colitis-associated colorectal cancer. High-throughput $16 \mathrm{~S}$ rRNA pyrosequencing was performed on tumoral (T) and chronically inflamed (NT) colonic specimens isolated from WT and Nod $2^{---}$animals $(n=4)$. $P$ values were determined by the Mann-Whitney $U$ test. (A) PCoA plot generated on nonabundance-based UniFrac distance matrices, (B) CCA based on observed genera. (C) Percentage of phyla and (D) top 10 genera, with abundance identified by bray-curtis model of SIMPER analysis. F, Firmicutes; B, Bacteroidetes; P, Proteobacteria. (E and F) Nonparametric quantification of microbiota diversity within (E) tumoral and (F) nontumoral tissues. Mean \pm SEM. ${ }^{*} P<0.05$.

$\mathrm{Nod}^{+/+}$animals differed in terms of the abundance of Escherichia/ Shigella, Robinsoniella, and Paludibacter - even though both group of animals were colitis vulnerable. This observation suggests that the latter changes in abundance were not correlated with the disease risk (Supplemental Figures 11 and 12). In contrast, the preponderance of Bacteroides was significantly greater in both WT $(P<0.01)$ and Nod2 $2^{--}(P<0.05)$ recipients recolonized with disease-predisposing microbiota, when compared with recipients recolonized with SPF WT microflora. Furthermore, Butyrivibrio and Lachnobacterium were significantly less preponderant in both WT recipients $(P<0.001$ and $P<0.02$, respectively) and $N o d 2^{-/-}$recipients $(P=0.01$ and $P<0.02$, respectively) upon recolonization with fecal microbiota of $\mathrm{Nod}^{-/-}$donor, when compared with $\mathrm{Nod}^{+/+} \rightarrow \mathrm{GF}-\mathrm{Nod} 2^{+/+}$animals. In summary, our results demonstrate that the disease-predisposing flora in $\mathrm{Nod}^{-1-}$ mice intrinsically sensitize the colonic mucosa to chemical insult prior to the onset of any disease - even in WT mice. Importantly, reciprocal transplantation of microbiota rescued Nod2 ${ }^{-/-}$mice from the disease risk.

\section{Discussion}

Given the intestinal epithelium's intimate relationship with the microbiome throughout the host's entire life, this structure is likely to coordinate immune homeostasis by fostering the stability and resilience of its coevolved microbiota. In the mouse, the absence of (a) Rag2 and T-bet, (b) the angiotensin-converting enzyme 2 (ACE2), or (c) the Nod-like receptor pyrin domaincontaining protein 6 is known to predispose to the emergence of transmissible, disease-predisposing commensals $(26,36,37)$. Understanding how the gut mucosa discriminates between disease-predisposing microorganisms and mutualists may provide insights into disease onset and tumor progression in genetically predisposed individuals and their relatives. Herein, we identified a hitherto unexpected protective function of the CD-predisposing Nod2 gene: the orchestration of concerted, protective gut microbial communities against intestinal inflammation and tumorigenesis in mice. In fact, WT mice that were transiently cohoused with Nod2 $2^{-/-}$or RIP2 $2^{-/-}$mice showed a heightened susceptibility to col- 
A

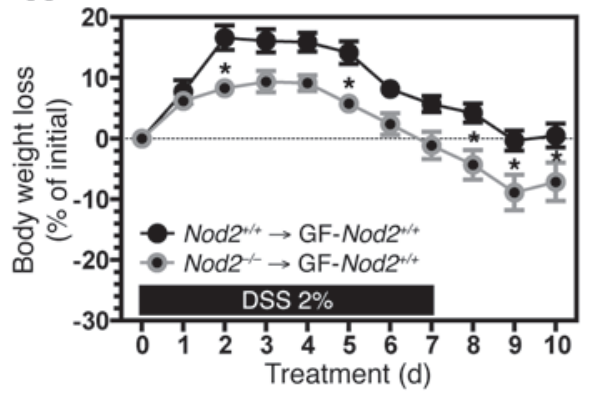

D

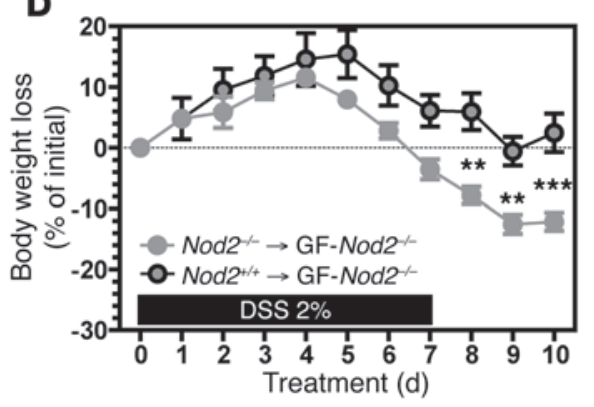

G

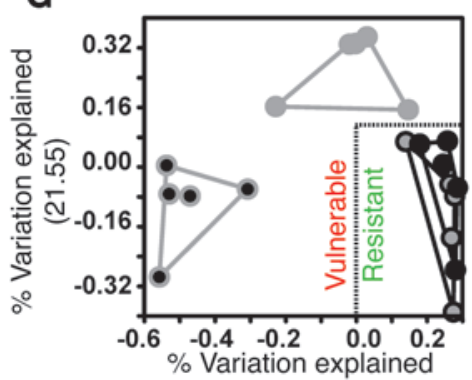

(52.58)
B
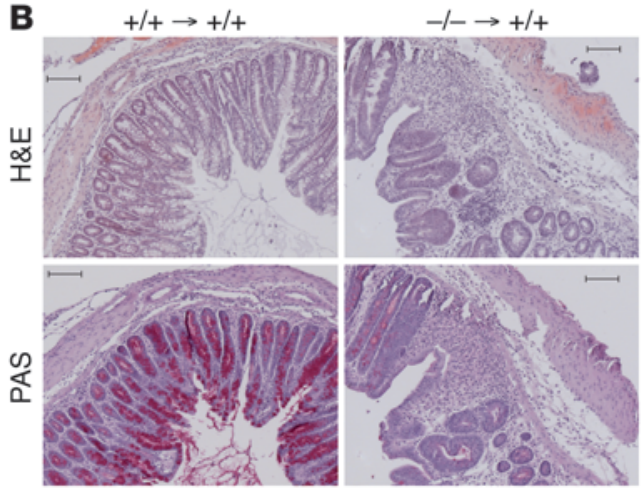

E

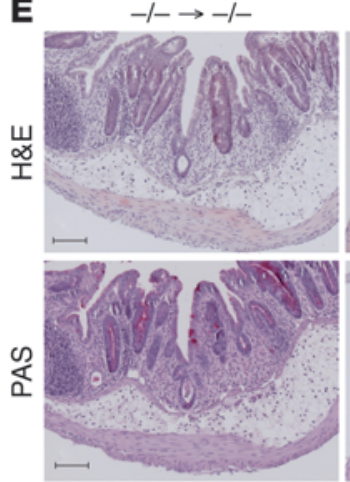

$-1-\rightarrow-1-$

$+/+\rightarrow-1-$

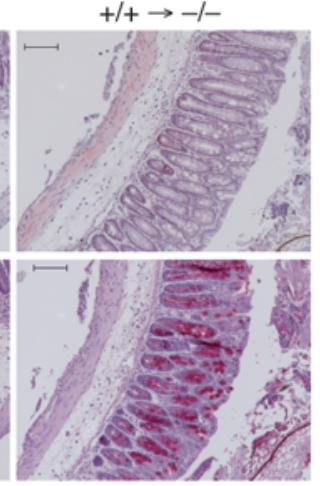

H

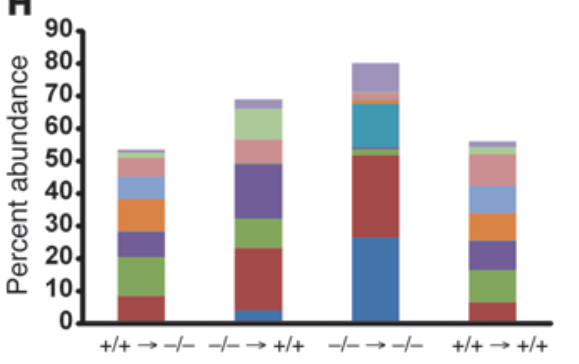

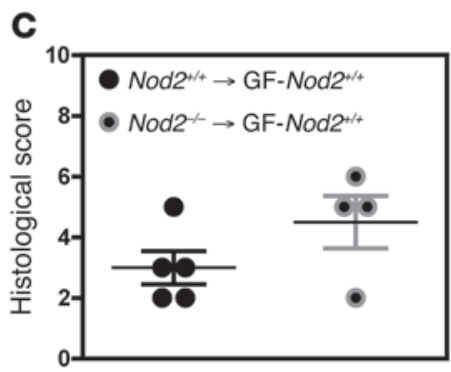

F

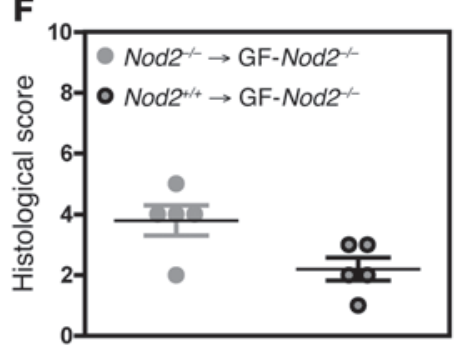

Figure 8

Dysbiotic microbial ecology of Nod2-deficient mice intrinsically instigates risk for colitis that is corrected by fecal transplantation. Four weeks before DSS-AOM challenge, GF-Nod2 ${ }^{--}(n=5)$ or GF-Nod2 $2^{+/}(n=5)$ mice were reconstituted with fecal microbiota from either Nod2 $2^{+/+}$or Nod $2^{-/-}$animals that were not exposed to DSS. (A and D) Changes in body weight were monitored daily. (B and E) Representative H\&E and PAS staining of paraffin-embedded transversal sections at day 10. Scale bar: $100 \mu \mathrm{m}$. (C and F) Histological scoring was evaluated at day 10. $(\mathbf{G}$ and $\mathbf{H})$ High-throughput 16S rRNA pyrosequencing was performed on fecal specimens isolated from all DSS-treated gnotobiotic animals $(n=5)$. (G) PCoA generated on nonabundance-based UniFrac distance matrices. $(\mathbf{H})$ Nonparametric quantification of microbiota diversity. $P$ values were determined by the Mann-Whitney $U$ test. Percentage of phyla abundance on top 10 genera was identified by bray-curtis model of SIMPER analysis. Mean \pm SEM. ${ }^{*} P<0.05 ;{ }^{* \star} P<0.01 ;{ }^{* * *} P<0.001$.

itis and CAC. This demonstrated that the changes in ecological niches created by NOD2 deficiency conferred a transmissible disease risk on mice - even WT animals. More importantly, abnormal expression of molecules involved in the epithelial barrier function was observed in WT animals upon cohousing, both during intestinal inflammation and prior to the experimental induction of colitis. This finding suggests that the microbiota of Nod2-deficient mice indeed sensitizes the colonic mucosa to injury. It is noteworthy that enhanced expression of claudin 5 (which is known to be involved in the pathogenesis of $\mathrm{CD}$; ref. 38) was observed in cohoused animals before and after injury. Although it could be argued that the observed changes were due (at least in part) to differences in the inflammatory state, it is important to bear in mind that GF animals recolonized with feces from nondiseased Nod2 $2^{--}$mice showed enhanced vulnerability to DSS (regardless of the recipient's NOD2 status). These observations suggested complex imprinting on the colonic mucosa via a transmissible dysbiosis that promotes disruption of the epithelial barrier function. Broad-spectrum antibiotic treatment abrogated the elevated sensitivity of both WT and Nod2-deficient mice to DSS-induced intestinal inflammation and tumorigenesis. Accordingly, a recent study highlighted a significant role for CD-associated NOD2 L1007fsins $C$ in the development of gut microbiota $(22,24)$. We next used a culture-independent approach to characterize changes 
in the composition of the microbial consortia. The risk of communicating disease to WT animals was primarily linked to changes in Bacteroides, Butyrivibrio, and Lachnobacterium communities - all of which may be important in the pathogenesis of human diseases (39). Notably, some of the aforementioned genera are involved in metabolism of indigestible dietary polysaccharides (40). Furthermore, commensal Bacteroides species (but not colitis-enriched Enterobacteriaceae) have been identified as a key determinant of disease risk in conventionally raised, genetically predisposed hosts (41). Our findings therefore illustrate the need to functionally assess disease-predisposing capacity not only in a single commensal but also in combinations of commensals that may fail to negatively regulate inflammation and/or to outcompete pathobionts even in a nongenetically predisposed host. Furthermore, determining the metabolic properties of disease-predisposing sets of commensals may provide insights into how NOD2 prevents their expansion and/or adhesion. This paradigm will help select the most efficient combination of probiotic strains, independently of the CD-predisposing Nod2 alleles.

In this study, disease severity was linked to disruption of the epithelial barrier function in response to a dysbiotic microbiota that accelerated the progression of tumors in the absence of NOD2. These results further support the hypothesis whereby early changes in the gut microbiota may imprint otherwise protective immunological responses that, later in life, can trigger colorectal cancer in the context of a primary immunodeficiency $(42,43)$. These findings may be of clinical relevance, since loss-of-function mutations of NOD2 are associated with an enhanced risk of CD $(13,15)$ and colorectal cancer in humans $(44-46)$. The colonic mucosa of patients with CD is characterized by increased secretion of interleukin- 6 , the magnitude of which is a predictor of the endoscopic recurrence and is correlated with the disease severity $(47,48)$. Interleukin- 6 regulates the differentiation and survival of epithelial cells by triggering activation of the associated Janus kinases and the STAT3 $(33,34)$. Upon injury, interleukin- 6 is primarily secreted by dendritic cells, which continuously inspect the luminal contents $(25,34)$. In line with previous findings $(34)$, we detected the expression of interleukin- 6 by dendritic-like cells and also colonocytes upon DSS challenge. Interleukin- 6 may well bind to its membrane receptor, which is restricted to a few cell populations (such as macrophages). Alternatively, after shedding of the interleukin- 6 receptor as a soluble form, interleukin- 6 may trans-signal to virtually all cell types by binding to a soluble cytokine-receptor complex (49), because the coreceptor gp130 is expressed ubiquitously (50). Recent studies have also highlighted the key impact of interleukin- 6 on tumor progression within chronically inflamed lesions. Importantly, Il6- or Stat3-knockout mice exhibited a lower tumor load and slower tumor growth in a model of CAC (28), whereas hyperactivation of these 2 genes promotes tumor growth $(33,34)$. It is noteworthy that the NOD2 genotype and anti-interleukin- 6 receptor therapy were found to strongly influence complex changes in the overall structure of the disease-predisposing mucosal- and fecal-associated flora. Future clinical and metagenomics studies should investigate the contribution of the complete set of genetic variants in known CD and CAC risk genes to the overall control of potentially dysbiotic microbial communities. This type of knowledge should facilitate the development of modulating therapies that seek to restore mutualistic interactions between the microbiota and the vulnerable intestinal barrier in patients with CD.

\section{Methods}

Colitis and colitis-associated cancer. Age- and gender-matched animals were housed 5 or 10 per cage for single-housing and cohousing experiments, respectively. In a half-day light cycle exposure and temperature-controlled SPF environment, as determined by the FELASA recommendations, animals had free access to a standard laboratory chow diet. Experimental models were performed as previously described (51). A cocktail combining streptomycin (Panpharma; $2 \mathrm{~g} / \mathrm{l}$ ), gentamicin (Panpharma; $200 \mathrm{mg} / \mathrm{l}$ ), ciprofloxacin (Panpharma; $125 \mu \mathrm{g} / \mathrm{l}$ ), and bacitracin (Acros Organics; $1 \mathrm{~g} / \mathrm{l}$ ) was used. Neutralization was performed by i.p. injecting $1 \mathrm{mg}$ MR16.1 (Chugai Pharmaceuticals Co Ltd.) per mouse. Mouse endoscopy was performed using the Coloview high-resolution system (Karl-Storz).

Recolonization. Nod2 ${ }^{-/-}$GF mice were generated at TAAM-CNRS and were transferred into autoclaved sterile microisolator cages before gavage with $200 \mu \mathrm{l}$ of fecal homogenates as indicated above ( 5 mice per cage). The remaining homogenate was kept frozen until processing for bacterial composition analysis.

Gene expression. Microarrays analysis was performed on distal colonic specimens as previously described (Agilent Technologies) (51). Differentially expressed genes (GEO accession numbers GSE32421 and GSE 36481) were filtered for a $P$ value of less than 0.01 by using moderated $t$ statistic with empirical Bayes shrinkage of SEM. Statistics were corrected for multiple testing using a false discovery rate approach. Gene ontology analysis was performed on all genes referenced in the UniGene database (52) by using the third hierarchical level of the ontology tree (Ingenuity Pathway Analysis). qRT-PCR analysis was performed as previously described (51).

Histopathological, immunohistochemistry, and FACS analysis. Paraffin-embedded 5 - $\mu \mathrm{m}$ sections of formalin-fixed colon specimens were stained with H\&E or PAS. The slides were blindly examined by two investigators with an AxioPlan2 microscope (Zeiss). Colonic lamina propria cells were isolated as previously described (53) and analyzed on a BD Calibur II cytometer. The following primary antibodies were used: anti-Ly6G PE-Cy7 (clone 1A8), anti-CD11b PerCP Cy5.5 (clone M1/70), anti-CD11c APC (clone HL3), anti-IA/IE FITC (clone 2G9), and Fc block (anti-CD32/CD16) (BD Pharmingen and R\&D Systems).

For immunofluorescence staining, colonic segments were embedded in Cryomatrix (Shandon, Thermo Scientific) and frozen on dry ice. Cryosections $(10 \mu \mathrm{m})$ were fixed for 5 minutes in $4 \%$ paraformaldehyde in PBS, washed in PBS- $0.05 \%$ Tween 20 (PBT), and incubated for 30 minutes at room temperature in PBT-5\% normal goat serum (saturation buffer) and then overnight at $4{ }^{\circ} \mathrm{C}$ with primary antibodies diluted in saturation buffer. Sections were washed in PBT and incubated for 1 hour with secondary antibodies. Nuclei were counterstained with 4',6-diamidino-2-phenylindole. Slides were mounted in Aqua Poly/Mount (PolySciences). The following antibodies were used: anti-interleukin-6 (M-19, sc-1265, Santa Cruz Biotechnology Inc.) and anti-CD4 (GK1.5), anti-CD8a (53-6.7), and biotin-conjugated anti-CD11b (M1/70) (all from BD Pharmingen). The secondary antibodies used were as follows: Alexa Fluor 488-conjugated anti-rabbit IgG, Alexa Fluor 594-conjugated anti-rat (Molecular Probes), and Cy3-conjugated streptavidin (Jackson ImmunoResearch Laboratories).

For immunohistochemistry, $5-\mu \mathrm{m}$ sections were deparaffinized and processed for antigen retrieval using $10 \mathrm{mM}$ citrate buffer ( $\mathrm{pH}$ 6), washed with PBS, incubated for 5 minutes in PBS- $3 \% \mathrm{H}_{2} \mathrm{O}_{2}$, washed in PBT, and incubated for 30 minutes in saturation buffer and then overnight at $4{ }^{\circ} \mathrm{C}$ with polyclonal anti-interleukin- 6 antibody (M-19, sc-1265, Santa Cruz Biotechnology Inc.). Detection was performed with biotinylated secondary antibody and then avidin biotinylated peroxidase complex for 1 hour and revealed with 3, 3'-diaminobenzidine (DAB) (Vectastain Elite ABC kit and DAB, Vector Laboratories). Sections were counterstained with hematoxylin. 
$q-P C R$ and pyrosequencing analysis. Colonic and fecal samples were collected and stored frozen before DNA extraction for q-PCR analysis by using the Qiamp DNA Stool Kit (Qiagen) and Nucleospin Tissue Kit (Macherey-Nagel), respectively. Absolute numbers of Bacteroides were determined by q-PCR analysis on a Stratagene Mx3005P (Agilent Technologies) from serial dilutions of plasmid constructs with a reference $16 \mathrm{~S}$ rDNA sequence. Pyrosequencing of extracted DNA was performed as previously described (22). A total of 1,000 high-quality reads was generated from each of the biological replicates. The specific forward and reverse primers sequences are available at Supplemental Table 5. 16S rRNA gene sequences with at least $97 \%$ similarity were clustered into species level OTUs using average neighbor algorithm. Phylogenetic affiliation of each OTU was governed by using RDP taxonomy and template file. PCoA and CCA were performed to visualize interactions among the microbial composition and structure, the host genotype, the anti-interleukin- 6 receptor treatment, and the presence of tumor. Phylogenetic-based (fast UniFrac) beta diversity was on phylogenetic tree generated by using FastTree version 2.0. Nonabundance-based UniFrac matrices were calculated and visualized using PCoA. PAST software was used for data visualization and for performing abundance-based PCoA, CCA, and SIMPER analysis on the bacterial genera observed in pyrosequencing data. Divergence-based phylogenetic diversity was calculated by phylo.diversity command implemented in mothur.

Cluster generation. OTUs were selected to contribute to the separation between WT and Nod2-deficient animals using the Mann-Whitney $U$ test, with subsequent selection of the 20 best $P$ values. In parallel, OTUs that were present in less than $50 \%$ of all samples were excluded from the cluster analysis. Prior to clustering, OTU abundances were normalized using $z$-score normalization to make OTUs comparable. Clustering was performed with TIBCO Spotfire (Integromics Biomarker Discovery), applying the unweighted pair group method using arithmetic means of the pairwise distances as a clustering method and correlation as a distance measure.

Statistics. The nonparametric Kruskal-Wallis or the parametric 1-way ANOVA test with multiple Dunn's comparison was used (GraphPad Software). Values are expressed as mean \pm SEM. Statistical significance was defined at a level of $P<0.05$.
Study approval. All animal studies were approved by the investigational review board of the Nord-Pas de Calais and by the University of Michigan's Committee on Use and Care of Animals. All animal experiments were performed in an accredited establishment (No. B59-108) according to governmental guidelines (No. 86/609/CEE).

\section{Acknowledgments}

Nod2 $2^{-/-}$and RIP2 $2^{-/-}$mice were provided by R.A. Flavell. We thank Karine Jambou, Estelle Douin, and Nathalie Froux for excellent technical assistance in maintaining mice colonies. A. Couturier-Maillard is a recipient of a PhD Fellowship from Inserm and region Nord-Pas de Calais. S. Normand is a recipient of a Postdoctoral Fellowship from the Ligue contre le Cancer. This work was supported by grants from the FRM (to B. Ryffel and M. Chamaillard), the ARC (to M. Chamaillard), the European Union-FEDER (to M. Chamaillard and B. Ryffel), Le Studium (to B. Ryffel), the DFG Cluster of Excellence Inflammation at Interfaces (to S. Schreiber and P. Rosenstiel), the BMBF Network Chronic Inflammatory Diseases (to P. Rosenstiel), and DFG Ro 2994-5-1 (to P. Rosenstiel).

Received for publication December 2, 2011, and accepted in revised form November 1, 2012.

Address correspondence to: Mathias Chamaillard, 1 rue du Professeur Calmette, BP 245, 59019 Lille Cedex, France. Phone: 33.0.359.31.74.27; Fax: 33.0.359.31.74.80; E-mail: mathias. chamaillard@inserm.fr.

Thomas Secher's present address is: Institut National de la Santé et de la Recherche Médicale, Unité Mixte de Recherche 1043, Toulouse, France.

Ateequr Rehman's present address is: Universitätsklinikum Freiburg, Freiburg, Germany.
1. Arumugam M, et al. Enterotypes of the human gut microbiome. Nature. 2011;473(7346):174-180.

2. Ley RE, et al. Evolution of mammals and their gut microbes. Science. 2008;320(5883):1647-1651.

3. Clarke TB, Davis KM, Lysenko ES, Zhou AY, Yu $\mathrm{Y}$, Weiser JN. Recognition of peptidoglycan from the microbiota by Nod 1 enhances systemic innate immunity. Nat Med. 2010;16(2):228-231.

4. Garrett WS, Gordon JI, Glimcher LH. Homeostasis and inflammation in the intestine. Cell. 2010; 140(6):859-870.

5. Kandori H, Hirayama K, Takeda M, Doi K. Histochemical, lectin-histochemical and morphometrical characteristics of intestinal goblet cells of germfree and conventional mice. Exp Anim. 1996; 45(2):155-160.

6. Pabst $\mathrm{O}$, et al. Adaptation of solitary intestinal lymphoid tissue in response to microbiota and chemokine receptor CCR7 signaling. J Immunol. 2006;177(10):6824-6832.

7. Atarashi $\mathrm{K}$, et al. Induction of colonic regulatory $\mathrm{T}$ cells by indigenous Clostridium species. Science. 2011;331(6015):337-341.

8. Asquith M, Powrie F. An innately dangerous balancing act: intestinal homeostasis, inflammation, and colitis-associated cancer. J Exp Med. 2010; 207(8):1573-1577.

9. Scanlan PD, Shanahan F, O'Mahony C, Marchesi JR. Culture-independent analyses of temporal variation of the dominant fecal microbiota and targeted bacterial subgroups in Crohn's disease.
J Clin Microbiol. 2006;44(11):3980-3988.

10. Manichanh C, et al. Reduced diversity of faecal microbiota in Crohn's disease revealed by a metagenomic approach. Gut. 2006;55(2):205-211.

11. Joossens M, et al. Dysbiosis of the faecal microbiota in patients with Crohn's disease and their unaffected relatives. Gut. 2011;60(5):631-637.

12. Terzic J, Grivennikov S, Karin E, Karin M. Inflammation and colon cancer. Gastroenterology. 2010;138(6):2101-2114.e5.

13. Hugot JP, et al. Association of NOD2 leucine-rich repeat variants with susceptibility to Crohn's disease. Nature. 2001;411(6837):599-603.

14. Hampe $J$, et al. Association between insertion mutation in NOD2 gene and Crohn's disease in German and British populations. Lancet. 2001; 357(9272):1925-1928.

15. Ogura Y, et al. A frameshift mutation in NOD2 associated with susceptibility to Crohn's disease. Nature. 2001;411(6837):603-606.

16. Watanabe T, et al. Muramyl dipeptide activation of nucleotide-binding oligomerization domain 2 protects mice from experimental colitis. J Clin Invest. 2008;118(2):545-559.

17. Kobayashi KS, et al. Nod2-dependent regulation of innate and adaptive immunity in the intestinal tract. Science. 2005;307(5710):731-734.

18. Geddes $\mathrm{K}$, et al. Identification of an innate $\mathrm{T}$ helper type 17 response to intestinal bacterial pathogens. Nat Med. 2011;17(7):837-844.

19. Kim YG, Park JH, Shaw MH, Franchi L, Inohara N,
Nunez G. The cytosolic sensors Nod1 and Nod2 are critical for bacterial recognition and host defense after exposure to Toll-like receptor ligands. Immunity. 2008;28(2):246-257.

20. Chamaillard M, Girardin SE, Viala J, Philpott DJ. Nods, Nalps and Naip: intracellular regulators of bacterial-induced inflammation. Cell Microbiol. 2003;5(9):581-592.

21. Petnicki-Ocwieja $\mathrm{T}$, et al. Nod2 is required for the regulation of commensal microbiota in the intestine. Proc Natl Acad Sci U S A. 2009;106(37):15813-15818.

22. Rehman A, et al. Nod2 is essential for temporal development of intestinal microbial communities. Gut. 2011;60(10):1354-1362.

23. Mondot $S$, et al. Altered gut microbiota composition in immune-impaired Nod2(-/-) mice. Gut. 2012; 61(4):634-635.

24. Frank DN, et al. Disease phenotype and genotype are associated with shifts in intestinal-associated microbiota in inflammatory bowel diseases. Inflamm Bowel Dis. 2011;17(1):179-184.

25. Denning TL, Wang YC, Patel SR, Williams IR, Pulendran B. Lamina propria macrophages and dendritic cells differentially induce regulatory and interleukin 17-producing $\mathrm{T}$ cell responses. Nat Immunol. 2007;8(10):1086-1094.

26. Garrett WS, et al. Communicable ulcerative colitis induced by T-bet deficiency in the innate immune system. Cell. 2007;131(1):33-45.

27. Lieu HT, et al. Reg2 inactivation increases sensitivity to Fas hepatotoxicity and delays liver regener- 
ation post-hepatectomy in mice. Hepatology. 2006; 44(6):1452-1464

28. Pickert G, et al. STAT3 links IL-22 signaling in intestinal epithelial cells to mucosal wound healing. J Exp Med. 2009;206(7):1465-1472.

29. Feagins LA, Souza RF, Spechler SJ. Carcinogenesis in IBD: potential targets for the prevention of colorectal cancer. Nat Rev Gastroenterol Hepatol. 2009; 6(5):297-305.

30. Rosenstiel P, et al. Influence of polymorphisms in the NOD1/CARD4 and NOD2/CARD15 genes on the clinical outcome of Helicobacter pylori infection. Cell Microbiol. 2006;8(7):1188-1198.

31. Mockelmann $\mathrm{N}$, et al. Investigation of innate immunity genes CARD4, CARD8 and CARD15 as germline susceptibility factors for colorectal cancer. BMC Gastroenterol. 2009;9:79.

32. Neufert C, Becker C, Neurath MF. An inducible mouse model of colon carcinogenesis for the analysis of sporadic and inflammation-driven tumor progression. Nat Protoc. 2007;2(8):1998-2004.

33. Bollrath J, et al. gp 130 -mediated Stat 3 activation in enterocytes regulates cell survival and cell-cycle progression during colitis-associated tumorigenesis. Cancer Cell. 2009;15(2):91-102.

34. Grivennikov S, et al. IL- 6 and stat 3 are required for survival of intestinal epithelial cells and development of colitis-associated cancer. Cancer Cell. 2009; 15(2):103-113.

35. Yamamoto M, Yoshizaki K, Kishimoto T, Ito H. IL-6 is required for the development of Th1 cell-mediated murine colitis. J Immunol. 2000;164(9):4878-4882.

36. Elinav E, et al. NLRP6 inflammasome regulates colonic microbial ecology and risk for colitis. Cell. 2011;145(5):745-757.

37. Hashimoto T, et al. ACE2 links amino acid malnutrition to microbial ecology and intestinal inflammation. Nature. 2012;487(7408):477-481.

38. Zeissig $\mathrm{S}$, et al. Changes in expression and distribution of claudin 2, 5 and 8 lead to discontinuous tight junctions and barrier dysfunction in active Crohn's disease. Gut. 2007;56(1):61-72.

39. Sartor RB. Microbial influences in inflammatory bowel diseases. Gastroenterology. 2008;134(2):577-594.

40. Backhed F, Ley RE, Sonnenburg JL, Peterson DA, Gordon JI. Host-bacterial mutualism in the human intestine. Science. 2005;307(5717):1915-1920.

41. Bloom SM, et al. Commensal Bacteroides species induce colitis in host-genotype-specific fashion in a mouse model of inflammatory bowel disease. Cell Host Microbe. 2011;9(5):390-403.

42. Secher T, Gaillot O, Ryffel B, Chamaillard M. Remote control of intestinal tumorigenesis by innate immunity. Cancer Res. 2010;70(5):1749-1752.

43. Olszak T, et al. Microbial exposure during early life has persistent effects on natural killer $T$ cell function. Science. 2012;336(6080):489-493.

44. Kurzawski G, et al. The NOD2 3020insC mutation and the risk of colorectal cancer. Cancer Res. 2004; 64(5):1604-1606.

45. Papaconstantinou I, et al. Association between mutations in the CARD15/NOD2 gene and colorectal cancer in a Greek population. Int J Cancer. 2005; 114(3):433-435.

46. Roberts RL, Gearry RB, Allington MD, Morrin HR, Robinson BA, Frizelle FA. Caspase recruit- ment domain-containing protein 15 mutations in patients with colorectal cancer. Cancer Res. 2006; 66(5):2532-2535.

47. Yamamoto T, Umegae S, Kitagawa T, Matsumoto $\mathrm{K}$. Mucosal cytokine production during remission after resection for Crohn's disease and its relationship to future relapse. Aliment Pharmacol Ther. 2004; 19(6):671-678.

48. Atreya R, et al. Blockade of interleukin 6 trans signaling suppresses $\mathrm{T}$-cell resistance against apoptosis in chronic intestinal inflammation: evidence in crohn disease and experimental colitis in vivo. Nat Med. 2000;6(5):583-588.

49. Jones SA, Scheller J, Rose-John S. Therapeutic strategies for the clinical blockade of IL-6/gp130 signaling. J Clin Invest. 2011;121(9):3375-3383.

50. Taga $T$, et al. Interleukin- 6 triggers the association of its receptor with a possible signal transducer, gp130. Cell. 1989;58(3):573-581.

51. Normand $S$, et al. Nod-like receptor pyrin domain-containing protein 6 (NLRP6) controls epithelial self-renewal and colorectal carcinogenesis upon injury. Proc Natl Acad Sci U S A. 2011; 108(23):9601-9606.

52. Tavazoie S, Hughes JD, Campbell MJ, Cho RJ, Church GM. Systematic determination of genetic network architecture. Nat Genet. 1999; 22(3):281-285

53. Weigmann B, Tubbe I, Seidel D, Nicolaev A, Becker C, Neurath MF. Isolation and subsequent analysis of murine lamina propria mononuclear cells from colonic tissue. Nat Protoc. 2007; 2(10):2307-2311 